\title{
iTRAQ-based proteomic analysis reveals potential regulatory networks in dust mite-related asthma treated with subcutaneous allergen immunotherapy
}

\author{
JUN BAI $^{1 *}$, JIA-YONG ZHONG ${ }^{2 *}$, WANG LIAO $^{1^{*}}$, RUO HU $^{3}$, \\ LIANG CHEN $^{1}$, XIAN-JIN WU ${ }^{4}$ and SHUANG-PING LIU ${ }^{5}$
}

\begin{abstract}
${ }^{1}$ Department of Pediatrics, Foshan Maternal and Children's Hospital Affiliated to Southern Medical University, Foshan, Guangdong 528000; ${ }^{2}$ Institute of Pediatrics, Guangzhou Women and Children's Medical Center, Guangzhou Medical University, Guangzhou, Guangdong 510623; ${ }^{3}$ School of Computer Science, Guangdong Polytechnic Normal University, Guangzhou, Guangdong 510000; ${ }^{4}$ Key Laboratory of Research and Utilization of Ethnomedicinal Plant Resources of Hunan Province, Key Laboratory of Hunan Higher Education for Western Hunan Medicinal Plant and Ethnobotany, College of Biological and Food Engineering, Huaihua University, Huaihua, Hunan 418008; ${ }^{5}$ Chronic Disease Research Center, Medical College, Dalian University, Dalian, Liaoning 116622, P.R. China
\end{abstract}

Received May 22, 2019; Accepted February 24, 2020

DOI: $10.3892 / \mathrm{mmr} .2020 .11472$

\begin{abstract}
Asthma is one of the most common childhood chronic diseases worldwide. Subcutaneous immunotherapy (SCIT) is commonly used in the treatment of house dust mite (HDM)-related asthma in children. However, the therapeutic mechanism of SCIT in asthma remains unclear. The present study aimed to investigate the molecular biomarkers associated with HDM-related asthma in asthmatic children prior and subsequent to SCIT treatment compared with those in healthy children via proteomic analysis. The study included a control group (30 healthy children), -Treatment group (30 children with HDM-related allergic asthma) and +Treatment group (30 children with HDM-related allergic asthma treated with SCIT). An isobaric labeling with relative and absolute quantification-based method was used to analyze serum
\end{abstract}

Correspondence to: Dr Shuang-Ping Liu, Chronic Disease Research Center, Medical College, Dalian University, 10 Xufu Street, Dalian, Liaoning 116622, P.R. China

E-mail: liuhshuangping@dlu.edu.cn

Dr Xian-Jin Wu, Key Laboratory of Research and Utilization of Ethnomedicinal Plant Resources of Hunan Province, Key Laboratory of Hunan Higher Education for Western Hunan Medicinal Plant and Ethnobotany, College of Biological and Food Engineering, Huaihua University, 180 Huaidong Road, Hecheng, Huaihua, Hunan 418008, P.R. China

E-mail: hhuxianjin@163.com

*Contributed equally

Key words: dust mite-related asthma, subcutaneous immunotherapy, isobaric tags for relative and absolute quantification proteome changes to detect differentially expressed proteins, while functional enrichment and protein-protein interaction network analysis were used to select candidate biomarkers. A total of 72 differentially expressed proteins were detected in the -Treatment, +Treatment and control groups. A total of 33 and 57 differentially expressed proteins were observed in the -Treatment vs. control and +Treatment vs. control groups, respectively. Through bioinformatics analysis, 5 candidate proteins [keratin 1 (KRT1), apolipoprotein B (APOB), fibronectin 1, antithrombin III (SERPINC1) and $\alpha$-1-antitrypsin (SERPINA1)] were selected for validation by western blotting; among them, 4 proteins (KRT1, APOB, SERPINC1 and SERPINA1) showed robust reproducibility in asthma and control samples. This study illustrated the changes in proteome regulation following SCIT treatment for asthma. The 4 identified proteins may serve as potential biomarkers prior and subsequent to SCIT treatment, and help elucidate the molecular regulation mechanisms of SCIT to treat HDM-related asthma.

\section{Introduction}

Asthma is a major public health problem affecting 300 million individuals worldwide, and its incidence is increasing annually (1). In 2013, the National Pediatric Asthma Collaborative Group conducted an epidemiological survey on children aged 0-14 years in 27 provinces and cities across China. It was identified that the prevalence of asthma among children in urban areas of China was $3.02 \%$, which is $50.6 \%$ higher compared with the prevalence in 2000 (2). Asthma is becoming an 'invisible killer' that seriously affects children's health. A total of $72.5 \%$ of asthmatic children have a history of allergic diseases (3). Pollen, house dust mites (HDMs), animal dander and fungi in the living environment are common allergens (4). HDMs are the most common aeroallergen in Asian countries (4). To manage allergic asthma, both avoidance measures 
and pharmacological treatments are used (5). However, due to the widespread existence of these allergens, it is very difficult to avoid them completely. In fact, asthma can be considered more as a syndrome as opposed to a single disease. Childhood asthma is usually mediated by a type 2 immune response (5). Type 2 immune-mediated (allergic) asthma is characterized by the presence of serum immunoglobulin $\mathrm{E}(\mathrm{IgE})$ antibodies and/or a positive skin-prick test for allergens (5). Allergen immunotherapy (AIT) is the only disease-modifying treatment option for patients with IgE-mediated inhalant allergies. Although it has been used in clinical practice for $>100$ years, most innovations in terms of AIT efficacy and safety have been developed in the last two decades. Major recent developments in AIT include subcutaneous immunotherapy (SCIT) and sublingual immunotherapy (6). SCIT involves the administration of gradually increasing quantities of an allergen vaccine to an allergic subject and inducing immunological tolerance to decrease symptom severity and decrease the need for pharmacotherapeutic interventions, ultimately improving the quality of life. SCIT is the only treatment that can change the natural course of allergic disease (6).

Although SCIT is now commonly used throughout the world and data for its efficacy are accumulating, certain patients do not respond to the treatment (7). There is no consensus on candidate surrogate biomarkers to predict its efficacy, or biomarker combinations that would be prognostic, predictive and/or surrogate for the clinical response to SCIT (8). The identification and validation of specific predictive biomarkers of the SCIT response is currently an active field of research and could improve the selection process and clinical management of patients receiving SCIT. Proteomics is a powerful method for studying new diagnostic and therapeutic targets. Owing to the rapid development of mass spectrometry (MS), the consistency of peptide identification and protein sequence coverage in complex biological samples has been significantly improved (9). Isobaric labeling with relative and absolute quantification (iTRAQ) is considered to be a reliable technique due to its high proteomic coverage and high labeling efficiency, particularly when information is not available from two-dimensional electrophoresis, and is widely used in quantitative proteomics (10). In the present study, iTRAQ-based proteomics was used to screen candidate biomarkers for HDM-associated asthma in order to understand the mechanism of the disease. The purpose of the present study was to provide a theoretical basis for the early clinical diagnosis of the disease and make early intervention possible .

The present study compared the serum proteomics of children with HDM-associated allergic asthma prior and subsequent to dust mite SCIT, and explored the molecular mechanisms of SCIT in the treatment of HDM-associated asthma, which could provide a comprehensive theoretical basis for more effective clinical treatment options.

\section{Materials and methods}

Study population. A total of 60 samples collected from 40 pediatric patients who met the inclusion criteria for HDM allergy-related asthma were enrolled. The patients included: A random sample from a group of 10 patients with HDM-related allergic asthma prior to SCIT therapy; a group of 10 patients with HDM-related allergic asthma following SCIT therapy; and a group of 20 patients with HDM-related allergic asthma before and after receiving SCIT therapy. A total of 30 ageand gender-matched normal children served as the control group. Patients were enrolled from the biobank of Foshan Maternal and Children's Hospital Affiliated to Southern Medical University between July 2016 and January 2019. The key inclusion criteria were as follows: Patients were diagnosed with mild or moderate allergic asthma according to the Global Initiative for Asthma (GINA) guidelines (10); and were between 4-14 years old. All patients were sensitized to HDM (Dermatophagoides pteronyssinus and Dermatophagoides farinae) allergens; this was measured by a positive skin prick test (SPT) and/or serum-specific IgE levels > class 2, and allergic asthma symptoms triggered by exposure to HDMs. Positivity to allergens on the SPT was determined when the size of the wheals caused by an allergen was $\geq$ the size of the wheals induced by histamine. A cutoff value of $0.35 \mathrm{kU} / 1$ for specific IgE was regarded as a positive result (class $1,0.35-0.7 \mathrm{kU} / \mathrm{l}$; class 2, 0.7-3.5 IU/1; class 3, 3.5-17.5 IU/1; class 4, 17.5-50 IU/1; class 5, 50-100 IU/1; and class 6, >100 IU/1). Patients were excluded if they had: i) Severe or uncontrolled asthma; ii) a specific IgE level of <0.7 IU/1 for any other allergic reasons, including cat/dog dander, Artemisia pollen and fungi; iii) a history of congenital cardiopulmonary diseases, malignant tumors, bronchiectasis, chronic rhinosinusitis or psychological or neurological diseases that may affect the outcome assessments; iv) received intercurrent treatment with $\beta$-blockers or angiotensin-converting enzyme inhibitors.

Among these patients, 30 children were first diagnosed with HDM-related allergic asthma without immunotherapy treatment, labeled as the -Treatment group, and 30 children exhibited well-controlled HDM-related allergic asthma after 3 years of SCIT treatment, and were labeled as the +Treatment group. Those patients that received the SCIT treatment also received control therapy such as inhalant corticosteroids, inhalant long-acting $\beta 2$ agonists, or antileukotrienes according to the GINA guidelines throughout the SCIT course. Asthma syndrome score (ASS), total medicine score (TMS), serum total (t)IgE, HDM specific (s)IgE, eosinophil count, the SPT and lung function were measured and recorded for each individual. TMS, total medicine score, ranging from 0 to 6 points, was assessed according to the total medicine intake on a 4-point scale: 0 , not taking medication; 1 , taking antihistamines or inhalant $\beta 2$ agonists; 2 , taking topical corticosteroids; and 3, taking oral corticosteroids.; ASS, asthma syndrome score was calculated as the total score of the four asthma symptoms and ranged from 0 to 12 points.

This study was approved by the Ethics Committee of Foshan Maternal and Children Hospital Affiliated to Southern Medical University(policy no. FSFY-MEC-2019-013). Written informed consent was obtained from the guardians of each participant.

Preparation of serum samples. Serum samples were processed to remove albumin and immunoglobulin $\mathrm{G}$ (IgG) using a ProteoPrep ${ }^{\circledR}$ Blue Albumin \& IgG Depletion kit (Sigma-Aldrich; Merck KGaA). The protein concentration of each was determined using a Bradford protein assay kit (Bio-Rad Laboratories, Inc.). Serum samples were then 
randomly assembled into two cohorts, the two cohorts were divided into three groups (control, -Treatment and +Treatment). Each group contained five samples in the first cohort and each group contained 25 samples in the second cohort. The first cohort was subsequently analyzed using iTRAQ ${ }^{\circledR}$ Reagent (SCIEX), and the second was analyzed by western blot analysis (Fig. 1).

Protein identification and analysis. ITRAQ tags were based on the manufacturer's protocols (SCIEX). Each protein sample was reduced by $100 \mu \mathrm{g}$ with Tris (2-carboxyethyl) phosphine hydrochloride reductant (SCIEX) at $60^{\circ} \mathrm{C}$ for $1 \mathrm{~h}$ and alkylated with methyl methanethiosulfonate cysteine blocker (SCIEX) at room temperature for $30 \mathrm{~min}$. Then, the protein was digested overnight by $2 \%$ trypsin (Promega Corporation) at $37^{\circ} \mathrm{C}$ at a ratio of 1:50 (enzymes: Substrates). Each sample was labeled with iTRAQ labels and then centrifuged in a vacuum centrifuge at a speed of $6,000 \mathrm{x} \mathrm{g}$ for $4 \mathrm{~h}$ at a temperature of $25^{\circ} \mathrm{C}$.

ITRAQ-labeled samples were first diluted to $100 \mu \mathrm{l}$ with a $\mathrm{H}_{2} \mathrm{O}$ buffer $\left(\mathrm{NH} 3+\mathrm{H}_{2} \mathrm{O}, \mathrm{pH}\right.$ 10.0) prior to high-performance liquid chromatography on a LC-20AD pump (Shimadzu Corporation) at $25^{\circ} \mathrm{C}$ on a Gemini ${ }^{\circledR} 3 \mu \mathrm{m} \mathrm{NX}-\mathrm{C} 18110 \mathrm{~A}$, $150 \times 2.00 \mathrm{~mm}$ Phenomenex column (from Phenomenex). The flow rate used for reversed-phase column separation was $0.2 \mathrm{ml} / \mathrm{min}$ with $\mathrm{H}_{2} \mathrm{O}$ (mobile phase A) and $80 \%$ acetonitrile (mobile phase B) with the following gradient system parameters: $5-10 \%$ B for 0-10 min; 10-40\% B for 10-60 min; 40-95\% $\mathrm{B}$ for $60-65 \mathrm{~min}$; and $95 \% \mathrm{~B}$ for $65-75 \mathrm{~min}$. Elution was monitored by absorbance at $214 / 280 \mathrm{~nm}$, and the fractions were collected every $50 \mathrm{sec}$; fractions were pooled for each sample and dehydrated by centrifugation at $6,000 \mathrm{x}$ g for $4 \mathrm{~h}$ at $25^{\circ} \mathrm{C}$ in vacuum centrifugation. Peptides were separated with mobile phase A ( $0.1 \%$ formic acid) and $5-40 \%$ mobile phase B $(0.1 \%$ formic acid and $80 \%$ acetonitrile) for $99 \mathrm{~min}(0.3 \mathrm{ml} / \mathrm{min}$ flow rate). MS analysis was performed on a $\mathrm{Q}$ Exactive $^{\mathrm{TM}}$ Hybrid Quadrupole-Orbitrap ${ }^{\mathrm{TM}}$ Mass Spectrometer (Thermo Fisher Scientific, Inc.) with the following parameters: MS spectra were acquired across the scan range of $350-1,800 \mathrm{~m} / \mathrm{z}$ in high-resolution mode $(>35,000)$, and $100 \mathrm{msec}$ was accumulated per spectrum. A maximum of 20 precursors per cycle were selected for fragmentation, with $120 \mathrm{msec}$ set as the minimum accumulation time for each precursor and $10 \mathrm{sec}$ for dynamic exclusion. A search for raw MS data was conducted against the human protein database using ProteinPilot ${ }^{\mathrm{TM}}$ Software 4.5 (SCIEX). The search parameters were set as 'cys alkylation' and 'methyl methanethiosulfonate', additionally 'digestion' and 'trypsin' were allowed; the false discovery rate was set to $<0.01$.

Western blot analysis. Proteins from the 25 samples of each group in cohort two were stored overnight with 4 times the volume acetone to obtain precipitate protein. The precipitate protein was then lysed in lysis buffer (Beyotime Institute of Biotechnology), and protein concentrations were measured using a Bradford protein assay kit (Bio-Rad Laboratories, Inc.). Following electrophoresis with $25 \mu \mathrm{g}$ protein/lane via 10-15\% SDS-PAGE, the separated proteins were transferred onto PVDF membranes (Sigma-Aldrich; Merck KGaA) and blocked with $5 \%$ skimmed milk for $2 \mathrm{~h}$ at room temperature. They were then incubated with primary antibodies $(1: 1,000$;

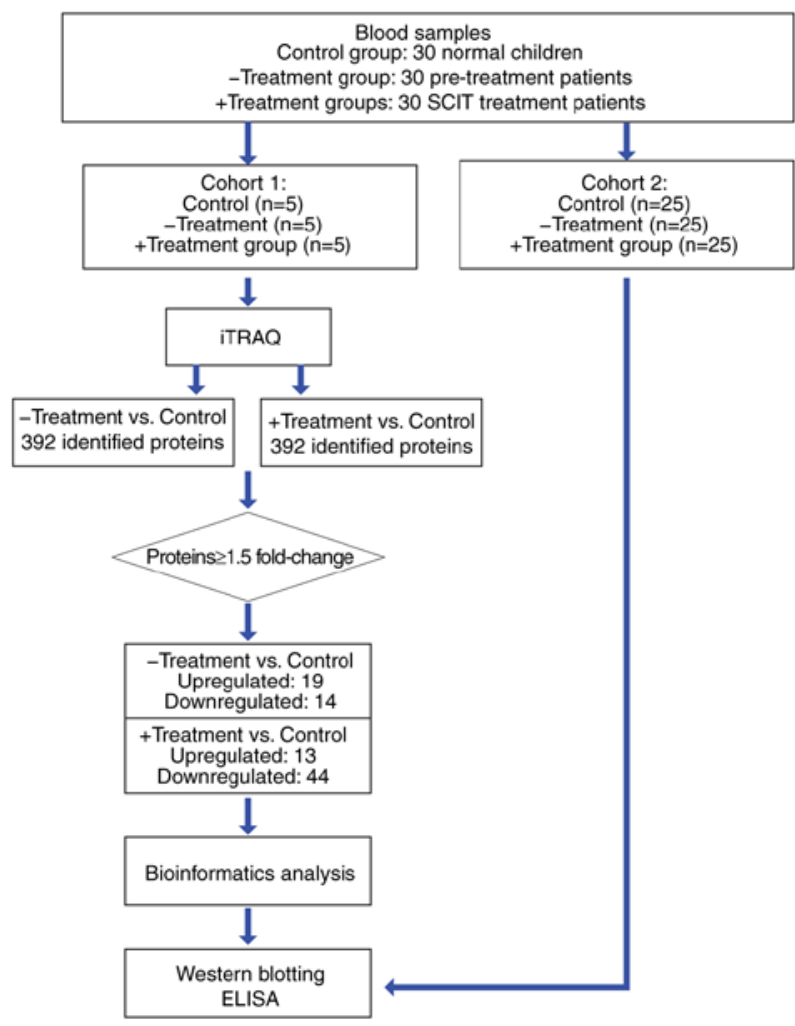

Figure 1. Experimental design and schematic workflow diagram. SCIT, subcutaneous immunotherapy; iTRAQ, isobaric tags for relative and absolute quantification.

all purchased from Abcam) against keratin (KRT)1 (cat. no. ab93652), apolipoprotein (APO)B (cat. no. ab27626), fibronectin 1 (FN1; cat. no. ab2413), antithrombin III (SERPINC1; cat. no. ab126598), $\alpha$-1-antitrypsin (SERPINA1; cat. no. ab207303) and GAPDH (cat. no. ab9485) overnight at $4^{\circ} \mathrm{C}$. Subsequently, horseradish peroxidase-conjugated goat anti-rabbit IgG (1:2,000; cat. no. ab6721; Abcam) was applied to the primary antibody-treated PVDF membranes at room temperature for $2 \mathrm{~h}$. The specific bands on these membranes were visualized using the SuperSignal ${ }^{\mathrm{TM}}$ chemiluminescent HRP substrates (Thermo Fisher Scientific, Inc.). Band intensities were quantitated using ImageJ software (version 2.0; National Institutes of Health).

ELISA. Serum concentrations of keratin 1 (KRT1; cat. no. KTE61888; Abbkine Scientific Co., Ltd.), apolipoprotein B (APOB; cat. no. KA1028; Abnova), fibronectin 1 (FN1; cat. no. KA3039; Abnova), antithrombin III (SERPINC1; cat. no. KA5093; Abnova) and $\alpha$-1-antitrypsin (SERPINA1; cat. no. KA0459; Abnova) of the 25 samples in each group were determined according to the manufacturers' protocols from the ELISA kits. Absorbance was measured at $450 \mathrm{~nm}$ using an ELISA reader. All samples were analyzed in triplicate, and the average concentration for each patient was calculated

Functional distribution analysis of differentially expressed proteins. Clustering of differentially expressed protein families and subfamilies was identified using the Protein analysis through evolutionary relationships (PANTHER) database (11-13), and analysis of functional Gene Ontology 
Table I. Characteristics values of the subjects in patients with HDM-related asthma and normal children.

\begin{tabular}{|c|c|c|c|c|c|c|}
\hline \multirow[b]{2}{*}{ Characteristics } & \multirow[b]{2}{*}{$\begin{array}{l}- \text { Treatment } \\
\quad(\mathrm{n}=30)\end{array}$} & \multirow[b]{2}{*}{$\begin{array}{l}+ \text { Treatment } \\
\quad(\mathrm{n}=30)\end{array}$} & \multirow[b]{2}{*}{$\begin{array}{l}\text { Normal } \\
\text { control } \\
(\mathrm{n}=30)\end{array}$} & \multicolumn{3}{|c|}{ P-value } \\
\hline & & & & $\begin{array}{l}\text {-Treatment vs. } \\
\text { +Treatment }\end{array}$ & $\begin{array}{l}\text {-Treatment } \\
\text { vs. Normal } \\
\text { control }\end{array}$ & $\begin{array}{l}\text { +Treatment } \\
\text { vs. Normal } \\
\text { control }\end{array}$ \\
\hline Sex, male/female, $n$ & $16 / 14$ & $15 / 15$ & $15 / 15$ & NA & NA & NA \\
\hline Age, median (IQR) years & $6(5-9)$ & $72(5-12)$ & $6(5-9)$ & 0.051 & 1.00 & 0.221 \\
\hline \multicolumn{7}{|c|}{ Laboratory values, median (IQR) } \\
\hline $\mathrm{EO}, 10^{9} / 1$ & $\begin{array}{c}0.65 \\
(0.11-0.91)\end{array}$ & $\begin{array}{c}0.33 \\
(0.10-0.47)\end{array}$ & $\begin{array}{c}0.21 \\
(0.10-0.36)\end{array}$ & 1.00 & 0.001 & 0.001 \\
\hline Total IgE, IU/ml & $\begin{array}{c}852.20 \\
(412.80-1,513.00)\end{array}$ & $\begin{array}{c}910.00 \\
(312.00-1,456.00)\end{array}$ & $\begin{array}{c}18.00 \\
(10.00-41.91)\end{array}$ & 1.00 & $<0.001$ & $<0.001$ \\
\hline $\begin{array}{l}\text { Specific IgE of } \\
\mathrm{HDM}, \mathrm{IU} / \mathrm{ml}\end{array}$ & $\begin{array}{c}85.00 \\
(35.00-200)\end{array}$ & $\begin{array}{c}70.00 \\
(35.00-145)\end{array}$ & 0 & 1.00 & $<0.001$ & $<0.001$ \\
\hline $\begin{array}{l}\text { Skin prick test of } \\
\mathrm{HDM}, \mathrm{cm}\end{array}$ & $\begin{array}{c}8.60 \\
(6.20-10.10)\end{array}$ & $\begin{array}{c}6.40 \\
(5.00-8.00)\end{array}$ & NA & $<0.001$ & NA & NA \\
\hline $\begin{array}{l}\text { Forced expiratory } \\
\text { volume in } 1 \mathrm{sec}, 1\end{array}$ & $\begin{array}{c}1.50 \\
(1.30-1.75)\end{array}$ & $\begin{array}{c}2.22 \\
(1.82-2.50)\end{array}$ & NA & $<0.001$ & NA & NA \\
\hline $\begin{array}{l}\text { Forced vital } \\
\text { capacity, } 1\end{array}$ & $\begin{array}{c}2.30 \\
(1.90-2.68)\end{array}$ & $\begin{array}{c}2.85 \\
(2.43-3.20)\end{array}$ & NA & $<0.001$ & NA & NA \\
\hline ASS & $\begin{array}{c}10.00 \\
(7.00-12.00)\end{array}$ & $\begin{array}{c}1.00 \\
(0.00-3.00)\end{array}$ & NA & $<0.001$ & NA & NA \\
\hline TMS & $3.0(1.00-3.0)$ & $0(0-0)$ & NA & $<0.001$ & NA & NA \\
\hline
\end{tabular}

IQR, interquartile range; EO, eosinophil count; IgE, immunoglobulin E; HDM, house dust mite; TMS, total medicine score; ASS, asthma syndrome score; NA, not applicable.

(GO) terms was performed with the online tool Metascape (http://metascape.org) (14). The enrichment-analyzed terms included 'biological processes', 'cellular components' and 'molecular functions'. In addition, a Kyoto Encyclopedia of Genes and Genomes (KEGG) pathways enrichment analysis was conducted (15-17). All genes in the genome were used as the enrichment background. Associations between proteins were also identified by using the protein-protein interaction (PPI) network generated from the Metascape database and visualized in Cytoscape (version 3.7.1) (18). A molecular complex detection (MCODE) algorithm was used by the Metascape tool to identify a densely connected network of protein-protein interactions.

Search Tool for the Retrieval of Interacting Genes/Proteins (STRING) PPI analysis. An association network of the identified proteins was established using the STRING database version 11 (19) (https://string-db.org) with the highest confidence interaction score (0.9). In addition, candidate proteins were selected according to their total PPI connection degrees, which is a rough representation of the importance of the network. These proteins may be the core proteins or key candidate genes that have significant physiological regulatory functions.

Statistical analysis. Data were analyzed using SPSS statistical software for Windows (version 19.0; IBM Corp.), and experimental non-parametric and parametric data were presented as median (interquartile range) or mean \pm standard deviation. Descriptive parameters were presented in Table I. Shapiro-Wilk normality test and Levene homogeneity test were used to assess the normal distribution and variance homogeneity of data. $\chi^{2}$ test was used for the comparison of categorical data (sex) in Table I (data not shown). Mann-Whitney U test (for two-group comparison of the skin prick test, FEV1, forced vital capacity, ASS and TMS values) or Kruskal-Wallis test followed by Dunn-Bonferroni post hoc method (for multi-group comparison of the age, EO, total $\mathrm{IgE}$ and specific $\operatorname{IgE}$ values) were applied to evaluate non-parametric values in Table I. The differences in protein expression levels of multiple groups (control group, -Treatment group and +Treatment groups) were analyzed using analysis of variance or Welch test, based on the results of the Levene test. Dunnett's test or Dunnett's T3 test was performed for post-hoc pairwise comparisons (control vs. -Treatment or control vs. +Treatment). For western blotting and ELISA validation, each group contained 25 samples, and each sample was repeated three times to obtain the mean value. $\mathrm{P}<0.05$ was considered to indicate a statistically significant difference.

\section{Results}

Characteristics of subjects. The clinical characteristics and demographic profiles of the patients with HDM-induced asthma and normal children are summarized in Table I. All subjects in each group were gender- and age-matched. Following SCIT treatment, 
A

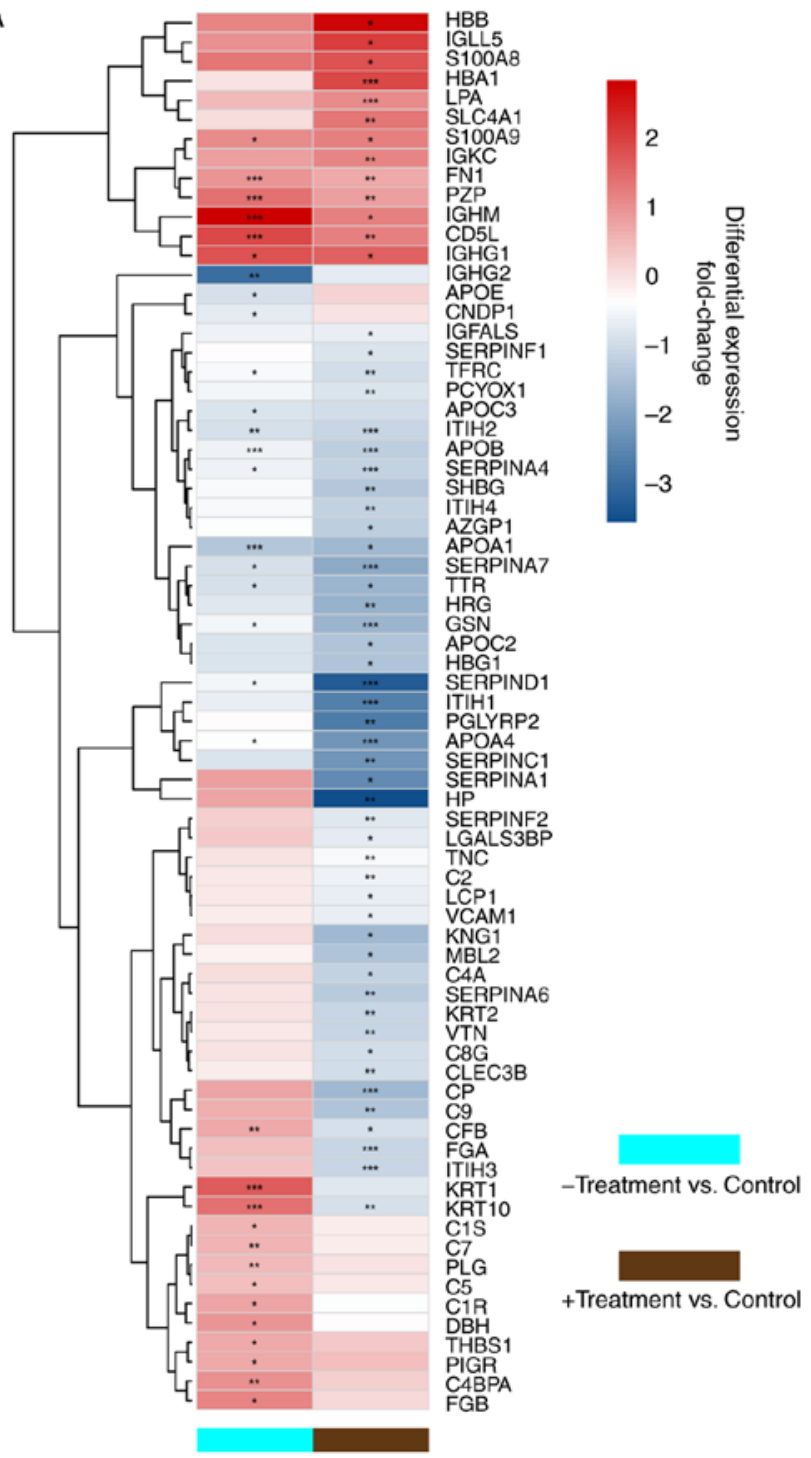

B
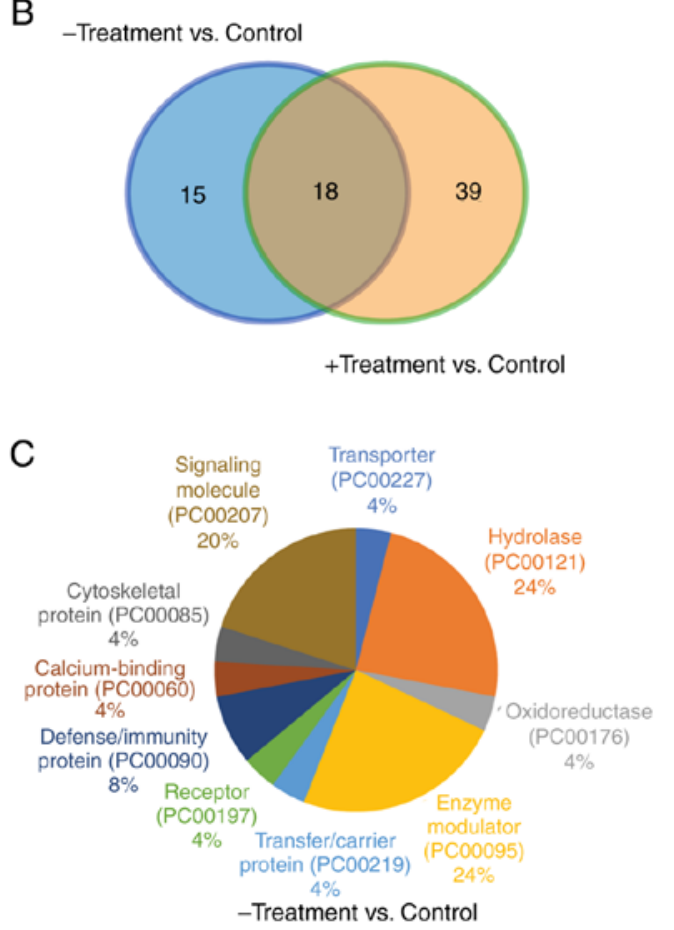

D

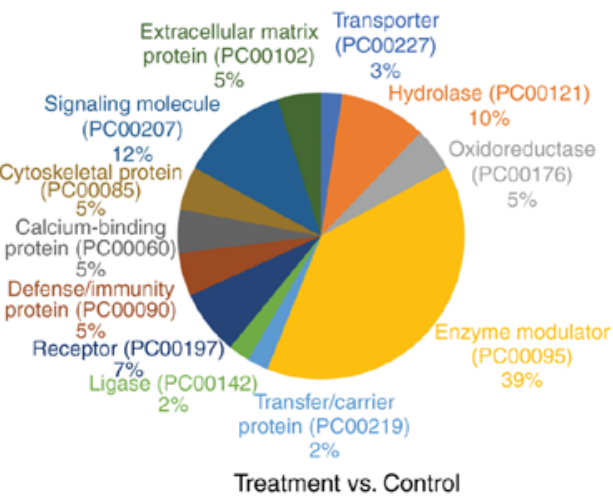

Figure 2. Heatmap, Venn diagram and classification of differentially expressed proteins from the -Treatment (pre-treatment) vs. control and + Treatment (following treatment) vs. control. (A) Normalized fold change in expression between the experimental groups and the control group are indicated as upregulated (red) or downregulated (blue) expression. The -Treatment vs. control comparison is indicated in light blue, while the +Treatment vs. control comparison is indicated in brown. ${ }^{*} \mathrm{P}<0.05,{ }^{* *} \mathrm{P}<0.01$ and ${ }^{* * *} \mathrm{P}<0.001$ vs. control. (B) Venn diagram showing the overlap of the significantly differentially expressed proteins in the -Treatment vs. control (blue) and +Treatment vs. control (orange) analyses. (C) Protein classes of the differentially expressed proteins in the -Treatment vs. control comparison as identified by the PANTHER database. (D) Protein classes of differentially expressed proteins in the -Treatment vs. control comparison as identified by the PANTHER database. PANTHER, Protein analysis through evolutionary relationships.

the patients with allergies exhibited a significant decrease in their symptoms, as measured by the ASS, which decreased from a score of 10.00 prior to treatment to 1.00 following treatment. Similarly, the use of medication significantly decreased the symptoms, as measured by TMS, which decreased from a score of 3.00 prior to treatment to 0.00 following SCIT treatment. The eosinophil counts were also significantly decreased by SCIT treatment. The levels of tIgE and SIgE were slightly, but not significantly, decreased from the beginning of treatment to 3 years after SCIT. Lung function parameters [forced expiratory volume in one second $\left(\mathrm{FEV}_{1 \%}\right)$ and forced vital capacity] demonstrated significant improvements following SCIT treatment.

Identification of differentially expressed proteins. Through iTRAQ technology, a total of 72 differentially expressed proteins were identified between the healthy children and
-Treatment or +Treatment patients (defined as fold change ratio $>1.5$ in control group vs. at least one in either the -Treatment or +Treatment groups), and the normalized fold change levels of these proteins are shown in a heatmap (Fig. 2A). A total of 33 (19 upregulated and 14 downregulated) and 57 (13 upregulated and 44 downregulated) significantly differently expressed proteins were identified in the -Treatment and +Treatment groups, respectively, compared with those in the control group (Tables II and III). A total of 18 overlapping differentially expressed proteins were identified in both groups, while 15 proteins were identified only in the -Treatment group and 39 proteins only in the + Treatment group (Fig. 2B).

Functional analysis of differentially expressed proteins. The differentially expressed proteins were characterized based on their functions. The protein class pie chart of the -Treatment 
Table II. Differentially expressed proteins identified between -Treatment samples and control samples using isobaric tags for relative and absolute quantitation technology.

\begin{tabular}{|c|c|c|c|c|c|}
\hline Number & Accession number & Gene name & Peptides average coverage rate, $\%$ & Fold change & P-value \\
\hline 1 & P01871 & IGHM & 87.86 & 7.31 & $1.09 \times 10^{-4}$ \\
\hline 2 & O43866 & CD5L & 80.4 & 3.84 & $4.89 \times 10^{-6}$ \\
\hline 3 & P01857 & IGHG1 & 88.79 & 3.37 & $3.03 \times 10^{-2}$ \\
\hline 4 & P04264 & KRT1 & 51.09 & 3.10 & $3.41 \times 10^{-7}$ \\
\hline 5 & P13645 & KRT10 & 51.88 & 2.58 & $3.22 \times 10^{-4}$ \\
\hline 6 & P20742 & $\mathrm{PZP}$ & 49.66 & 2.56 & $4.60 \times 10^{-5}$ \\
\hline 7 & P02675 & FGB & 29.33 & 2.19 & $3.56 \times 10^{-2}$ \\
\hline 8 & P06702 & S100A9 & 90.35 & 2.05 & $4.54 \times 10^{-2}$ \\
\hline 9 & P04003 & C4BPA & 63.32 & 2.01 & $8.82 \times 10^{-3}$ \\
\hline 10 & P02751 & FN1 & 57.04 & 1.89 & $1.31 \times 10^{-4}$ \\
\hline 11 & P09172 & DBH & 37.76 & 1.89 & $2.24 \times 10^{-2}$ \\
\hline 12 & P00736 & $\mathrm{C} 1 \mathrm{R}$ & 64.96 & 1.63 & $4.41 \times 10^{-2}$ \\
\hline 13 & P00751 & CFB & 68.19 & 1.61 & $7.29 \times 10^{-3}$ \\
\hline 14 & P07996 & THBS1 & 48.55 & 1.57 & $1.94 \times 10^{-2}$ \\
\hline 15 & P01833 & PIGR & 34.03 & 1.57 & $2.42 \times 10^{-2}$ \\
\hline 16 & P00747 & PLG & 81.36 & 1.56 & $9.69 \times 10^{-3}$ \\
\hline 17 & P10643 & $\mathrm{C} 7$ & 53.5 & 1.53 & $7.44 \times 10^{-3}$ \\
\hline 18 & P01031 & $\mathrm{C} 5$ & 52.21 & 1.52 & $1.58 \times 10^{-2}$ \\
\hline 19 & P09871 & $\mathrm{C} 1 \mathrm{~S}$ & 67.3 & 1.54 & $4.36 \times 10^{-2}$ \\
\hline 20 & P06727 & APOA4 & 89.39 & 0.62 & $4.01 \times 10^{-2}$ \\
\hline 21 & P06396 & GSN & 70.84 & 0.66 & $2.81 \times 10^{-2}$ \\
\hline 22 & P05546 & SERPIND1 & 70.34 & 0.66 & $1.03 \times 10^{-2}$ \\
\hline 23 & P04114 & APOB & 82.05 & 0.64 & $1.82 \times 10^{-6}$ \\
\hline 24 & P29622 & SERPINA4 & 58.08 & 0.63 & $2.24 \times 10^{-2}$ \\
\hline 25 & P02786 & TFRC & 40.79 & 0.60 & $4.47 \times 10^{-2}$ \\
\hline 26 & Q96KN2 & CNDP1 & 50.69 & 0.57 & $3.58 \times 10^{-2}$ \\
\hline 27 & P02656 & APOC3 & 79.8 & 0.53 & $3.64 \times 10^{-2}$ \\
\hline 28 & P05543 & SERPINA7 & 64.34 & 0.52 & $1.96 \times 10^{-2}$ \\
\hline 29 & P19823 & ITIH2 & 60.68 & 0.51 & $6.49 \times 10^{-3}$ \\
\hline 30 & P02649 & APOE & 89.59 & 0.51 & $1.73 \times 10^{-2}$ \\
\hline 31 & P02766 & TTR & 86.39 & 0.50 & $1.31 \times 10^{-2}$ \\
\hline 32 & P02647 & APOA1 & 94.01 & 0.37 & $2.13 \times 10^{-5}$ \\
\hline 33 & P01859 & IGHG2 & 85.58 & 0.12 & $6.22 \times 10^{-3}$ \\
\hline
\end{tabular}

vs. control group (Fig. 2C) demonstrates a high percentage of hydrolase, enzyme modulator and signaling molecule class proteins. In the +Treatment group (Fig. 2D), the percentages of hydrolase and signaling molecule class proteins were decreased, while the percentage of enzyme modulator class proteins was increased. These differences in protein class between the -Treatment and +Treatment groups suggested that enzyme modulator proteins potentially serve a major role in the treatment process and should be investigated in greater detail.

The enriched cellular components (blue), molecular functions (green) and biological processes (orange) GO terms for the proteins are presented in Fig. 3A. GO analysis revealed proteins mainly associated with blood microparticle and collagen-containing extracellular matrix (ECM). In addition, this analysis suggested that proteins were mainly enriched for biological processes associated with the acute inflammatory response and protein activation cascade (Fig. 3A). The top enriched KEGG pathways for the differentially expressed proteins included complement and coagulation cascades, systemic lupus erythematosus and Staphylococcus aureus infection (Fig. 3B).

According to the MCODE method, four sub-clusters of proteins were identified, as shown in Fig. 4B (MCODE1, MCODE2, MCODE3 and MCODE4); proteins in each cluster shared the same GO terms and KEGG pathways. Cluster MCODE1 included the proteins APOC2, APOE, APOB, APOA4, APOC3 and APOA1, which are associated with chylomicron remodeling (GO:0034371), chylomicron assembly (GO:0034378) and triglyceride-rich lipoprotein particle remodeling (GO:0034370). Cluster MCODE2 included complement component 5, vitronectin, complement $8 \gamma$, complement component 9 and complement 
Table III. Differentially expressed proteins identified between +Treatment samples and control samples using isobaric tags for relative and absolute quantitation technology.

\begin{tabular}{|c|c|c|c|c|c|}
\hline Number & Accession number & Gene name & Peptides average coverage rate, $\%$ & Fold change & P-value \\
\hline 1 & P68871 & HBB & 99.32 & 6.73 & $1.02 \times 10^{-2}$ \\
\hline 2 & B9A064 & IGLL5 & 63.08 & 4.06 & $3.94 \times 10^{-2}$ \\
\hline 3 & P69905 & HBA1 & 80.99 & 3.80 & $7.55 \times 10^{-5}$ \\
\hline 4 & P05109 & S100A8 & 65.59 & 3.44 & $1.29 \times 10^{-2}$ \\
\hline 5 & P01857 & IGHG1 & 88.79 & 2.99 & $2.51 \times 10^{-2}$ \\
\hline 6 & P02730 & SLC4A1 & 28.98 & 2.54 & $6.58 \times 10^{-3}$ \\
\hline 7 & P06702 & S100A9 & 90.35 & 2.33 & $1.44 \times 10^{-2}$ \\
\hline 8 & O43866 & CD5L & 80.40 & 2.31 & $3.38 \times 10^{-3}$ \\
\hline 9 & P01871 & IGHM & 87.86 & 2.25 & $3.20 \times 10^{-2}$ \\
\hline 10 & P01834 & IGKC & 98.13 & 2.15 & $3.76 \times 10^{-3}$ \\
\hline 11 & P08519 & LPA & 45.32 & 2.13 & $2.07 \times 10^{-4}$ \\
\hline 12 & P20742 & PZP & 49.66 & 1.75 & $9.19 \times 10^{-3}$ \\
\hline 13 & P02751 & FN1 & 57.04 & 1.57 & $9.39 \times 10^{-3}$ \\
\hline 14 & P06681 & $\mathrm{C} 2$ & 58.38 & 0.63 & $6.54 \times 10^{-3}$ \\
\hline 15 & P35858 & IGFALS & 55.54 & 0.61 & $2.13 \times 10^{-2}$ \\
\hline 16 & P19320 & VCAM1 & 31.12 & 0.61 & $4.17 \times 10^{-2}$ \\
\hline 17 & P13796 & LCP1 & 48.64 & 0.60 & $3.29 \times 10^{-2}$ \\
\hline 18 & P24821 & $\mathrm{TNC}$ & 16.45 & 0.59 & $1.69 \times 10^{-3}$ \\
\hline 19 & Q08380 & LGALS3BP & 47.01 & 0.59 & $3.36 \times 10^{-2}$ \\
\hline 20 & P08697 & SERPINF2 & 67.01 & 0.55 & $8.73 \times 10^{-3}$ \\
\hline 21 & P36955 & SERPINF1 & 70.57 & 0.54 & $1.02 \times 10^{-2}$ \\
\hline 22 & Q9UHG3 & PCYOX1 & 41.19 & 0.52 & $7.92 \times 10^{-3}$ \\
\hline 23 & P00751 & $\mathrm{CFB}$ & 68.19 & 0.52 & $1.90 \times 10^{-2}$ \\
\hline 24 & P13645 & KRT10 & 51.88 & 0.50 & $8.22 \times 10^{-3}$ \\
\hline 25 & P07360 & $\mathrm{C} 8 \mathrm{G}$ & 76.24 & 0.49 & $1.49 \times 10^{-2}$ \\
\hline 26 & P05452 & CLEC3B & 71.29 & 0.49 & $6.74 \times 10^{-3}$ \\
\hline 27 & P02786 & TFRC & 40.79 & 0.48 & $1.53 \times 10^{-3}$ \\
\hline 28 & P35908 & KRT2 & 49.92 & 0.45 & $4.68 \times 10^{-3}$ \\
\hline 29 & Q06033 & ITIH3 & 60.67 & 0.44 & $6.01 \times 10^{-4}$ \\
\hline 30 & P04004 & VTN & 46.86 & 0.44 & $8.08 \times 10^{-3}$ \\
\hline 31 & P19823 & ITIH2 & 60.68 & 0.43 & $4.55 \times 10^{-4}$ \\
\hline 32 & P02671 & FGA & 50.58 & 0.43 & $2.76 \times 10^{-5}$ \\
\hline 33 & P29622 & SERPINA4 & 58.08 & 0.43 & $8.52 \times 10^{-4}$ \\
\hline 34 & Q14624 & ITIH4 & 78.17 & 0.42 & $1.22 \times 10^{-3}$ \\
\hline 35 & P0C0L4 & $\mathrm{C} 4 \mathrm{~A}$ & 84.52 & 0.42 & $2.49 \times 10^{-2}$ \\
\hline 36 & $\mathrm{P} 25311$ & AZGP1 & 74.50 & 0.41 & $3.44 \times 10^{-2}$ \\
\hline 37 & P04114 & APOB & 82.05 & 0.39 & $1.15 \times 10^{-12}$ \\
\hline 38 & P08185 & SERPINA6 & 58.52 & 0.39 & $1.02 \times 10^{-3}$ \\
\hline 39 & P04278 & SHBG & 53.73 & 0.37 & $1.52 \times 10^{-3}$ \\
\hline 40 & P02748 & C9 & 52.77 & 0.36 & $7.84 \times 10^{-3}$ \\
\hline 41 & P69891 & HBG1 & 80.27 & 0.36 & $1.24 \times 10^{-1}$ \\
\hline 42 & P11226 & MBL2 & 52.42 & 0.35 & $1.18 \times 10^{-2}$ \\
\hline 43 & P02655 & APOC2 & 82.18 & 0.35 & $2.85 \times 10^{-2}$ \\
\hline 44 & P02647 & APOA1 & 94.01 & 0.31 & $1.84 \times 10^{-2}$ \\
\hline 45 & P01042 & KNG1 & 68.79 & 0.31 & $2.96 \times 10^{-2}$ \\
\hline 46 & P00450 & $\mathrm{CP}$ & 84.41 & 0.30 & $9.02 \times 10^{-5}$ \\
\hline 47 & P06396 & GSN & 70.84 & 0.30 & $1.49 \times 10^{-7}$ \\
\hline 48 & P02766 & TTR & 86.39 & 0.29 & $4.14 \times 10^{-2}$ \\
\hline 49 & P04196 & HRG & 63.43 & 0.28 & $5.48 \times 10^{-3}$ \\
\hline 50 & P05543 & SERPINA7 & 64.34 & 0.25 & $2.47 \times 10^{-4}$ \\
\hline 51 & P06727 & APOA4 & 89.39 & 0.20 & $1.45 \times 10^{-7}$ \\
\hline
\end{tabular}


Table III. Continued.

\begin{tabular}{lclccc}
\hline Number & Accession number & Gene name & Peptides average coverage rate, $\%$ & Fold change & P-value \\
\hline 52 & P01008 & SERPINC1 & 74.35 & 0.19 & $1.14 \times 10^{-3}$ \\
53 & P01009 & SERPINA1 & 93.54 & 0.17 & $1.75 \times 10^{-2}$ \\
54 & P19827 & ITIH1 & 63.67 & 0.15 & $4.77 \times 10^{-4}$ \\
55 & Q96PD5 & PGLYRP2 & 63.89 & 0.14 & $1.41 \times 10^{-3}$ \\
56 & P05546 & SERPIND1 & 70.34 & 0.09 & $1.24 \times 10^{-6}$ \\
57 & P00738 & HP & 92.61 & 0.08 & $3.00 \times 10^{-3}$ \\
\hline
\end{tabular}

A

A Blood microparticle-

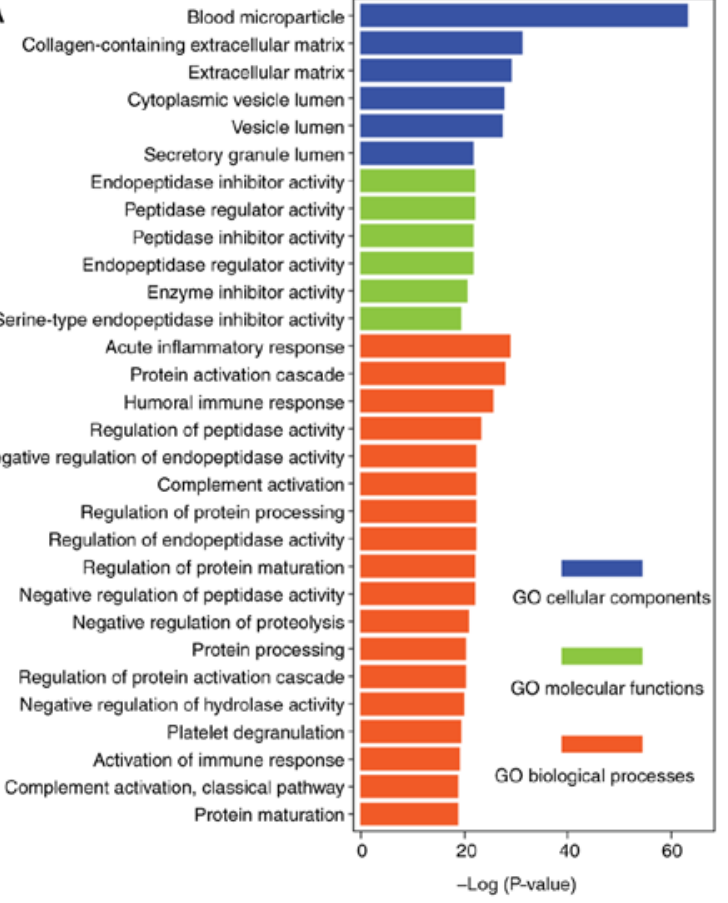

B

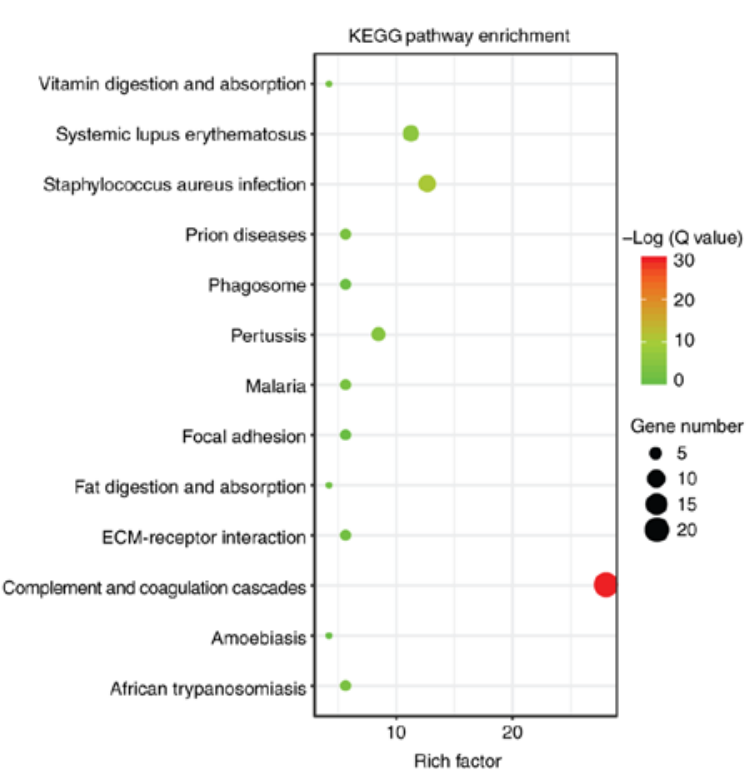

Figure 3. GO and KEGG enrichment analyses of differentially expressed proteins in the -Treatment vs. control and the +Treatment vs. control comparison determined using the Metascape database. (A) Using a negative log scale of accumulative hypergeometric P-values, the 30 most highly enriched GO terms are shown. (B) Scatter plot of enriched KEGG pathways for differentially expressed proteins. The color and size of the dots represent the range of Q-values in the negative log scale (corrected P-value) and gene number, respectively. GO, Gene Ontology; KEGG, Kyoto Encyclopedia of Genes and Genomes.

component 7 , which are associated with membrane attack complex (GO:0005579), complement and coagulation cascades (hsa04610), and complement activation and alternative pathway (GO:0006957). Cluster MCODE3 included KRT10, KRT2, KRT1 and vascular cell adhesion molecule 1, which are associated with structural constituent of epidermis (GO:0030280), peptide cross-linking (GO:0018149) and cornified envelope (GO:0001533). Finally, cluster MCODE4 included fibrinogen $\beta$ chain (FGB), lipoprotein A and FN1, which are involved in extracellular structure organization (GO:0043062). Further screening and analysis are required for these proteins.

STRING PPI network analysis. Next, PPI networks were constructed using STRING data concerning the differentially expressed proteins in the -Treatment (Fig. 5) and +Treatment groups (Fig. 6). These data allowed the major proteins to be determined in the PPI network. In the -Treatment vs. control analysis, 30/33 differentially expressed proteins were filtered to form the PPI network complex (Fig. 5A). The network contained 30 nodes and 34 edges. Among the 30 nodes, the top 10 most strongly connected PPI nodes were selected as hub proteins. These hub proteins were APOB, FN1, APOA1, APOE, SERPIND1, inter- $\alpha$-trypsin inhibitor heavy chain 2 (ITIH2), APOA4, FGB, APOC3 and plasminogen (Fig. 5B). APOB exhibited the highest network connectivity in this PPI network. In the +Treatment vs. control comparison, 54/57 differentially expressed proteins were filtered to form the PPI network complex (Fig. 6A). The PPI network contained 54 nodes and 117 edges. Among these nodes, the major hub proteins were APOA1, APOB, complement C4A (C4A), FGA, FN1, kininogen 1 (KNG1), SERPINA1, SERPINC1, ceruloplasmin and ITIH2 (Fig. 6B). Thus, APOB had the second highest degree of connectivity in this PPI network.

A more comprehensive PPI network complex was formed with protein nodes from all the differentially expressed proteins in both the -Treatment and +Treatment groups compared to the control group (Fig. S1). This comprehensive 

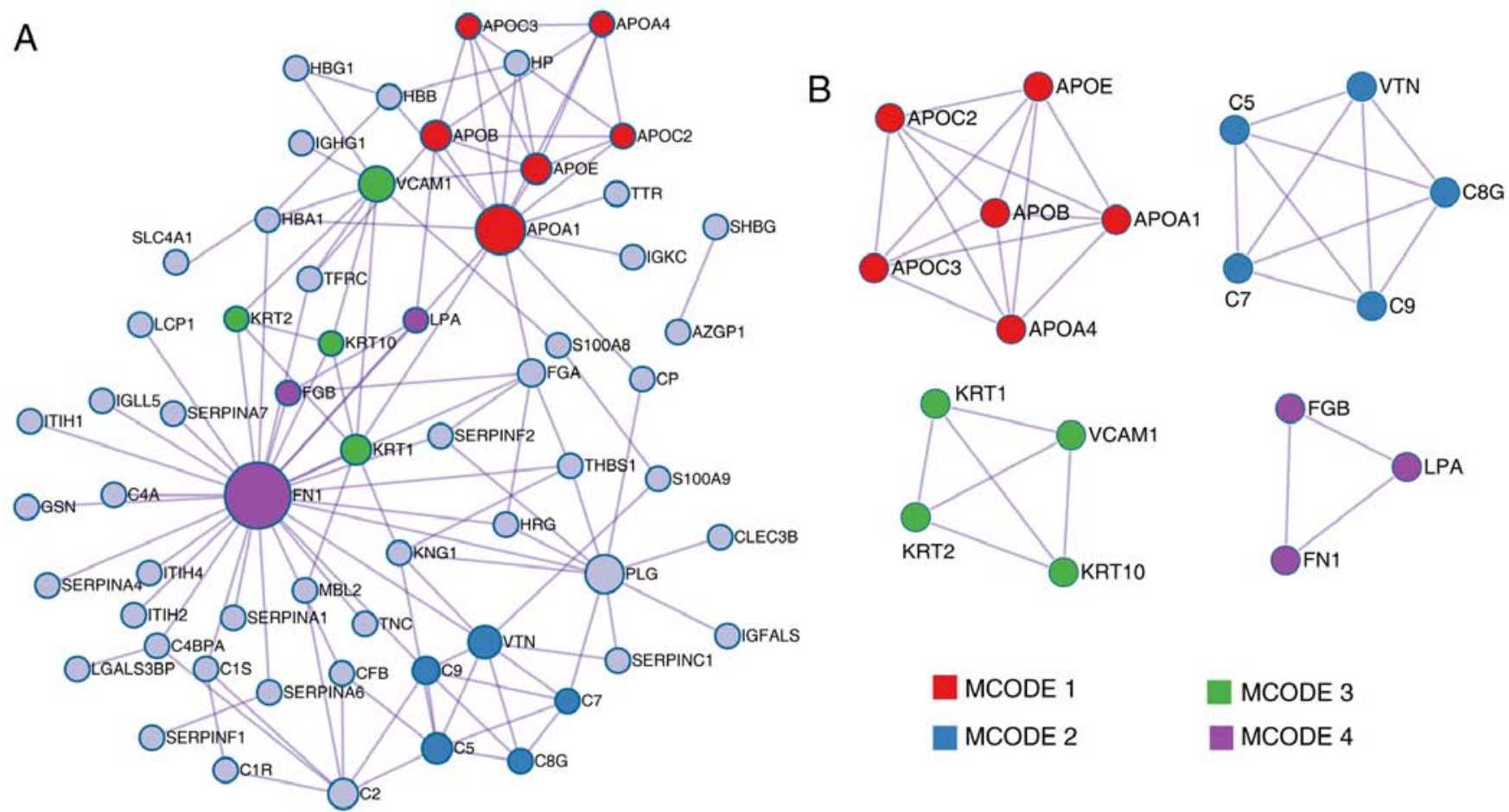

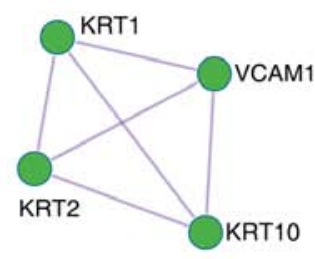

MCODE 1

MCODE 2

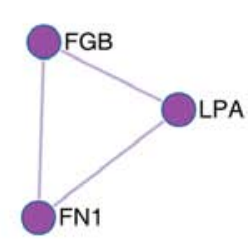

MCODE 3

MCODE 4

Figure 4. PPI network and MCODE components identified to be associated with the differentially expressed proteins from the -Treatment vs. control and +Treatment vs. control analyses. (A) PPI network analysis determined using Metascape. (B) Protein modules selected from the PPI using the MCODE method. Colors represent the different MCODE clusters. PPI, Protein-protein interaction; MCODE, Molecular Complex Detection.

A

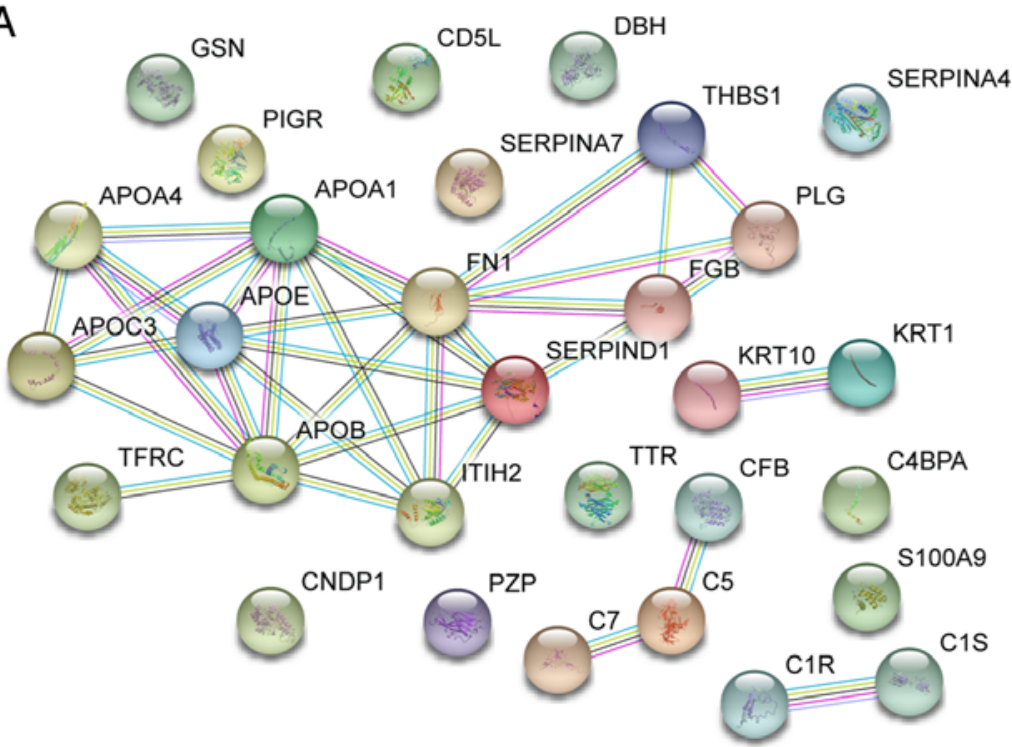

B

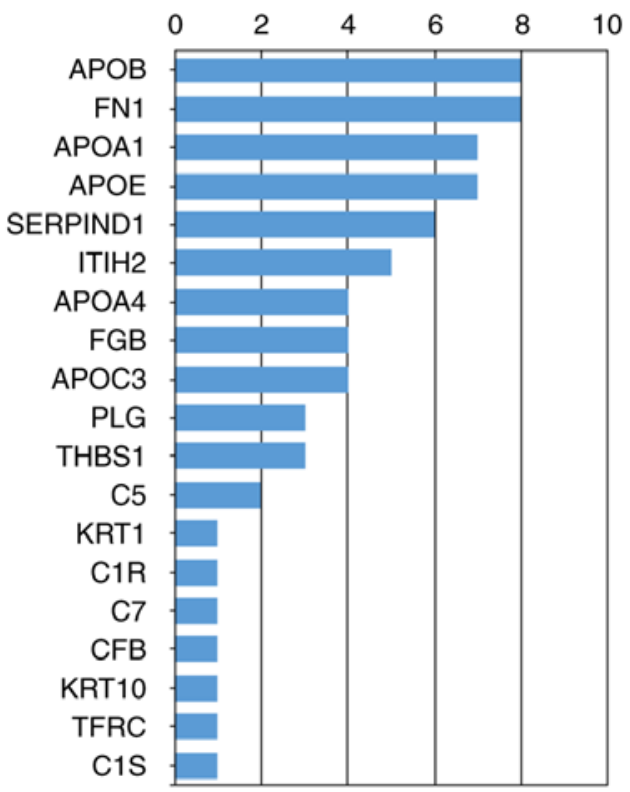

Figure 5. STRING analysis of PPI networks for the differentially expressed proteins from the -Treatment vs. control analysis. (A) PPI analysis of 33 differentially expressed proteins from -Treatment vs. control comparison according to the STRING database using a 'highest confidence' threshold (score of 0.9). The interactions between proteins are indicated by lines. (B) PPI network degree of the top 19 differentially expressed proteins. Network degree represents the total connections of one protein, which can be used as a rough indication of the centrality of the protein within the PPI networks. STRING, Search Tool for the Retrieval of Interacting Genes/Proteins; PPI, Protein-protein interaction.

network contained 68 nodes and 188 edges, and the top ten hub proteins were C4A, APOA1, FGA, FN1, KNG1, APOB, APOE, SERPINA1, SERPINC1 and SERPIND1. KRT1 was not a top PPI network connectivity protein, but was an MCODE hub protein (Fig. 4B) that was significantly differently expressed. Based on the above results, and as candidate proteins should exhibit strongly significant differences in expression, KRT1, APOB, FN1, SERPINC1 and SERPINA1 were selected as candidate proteins for further validation. 

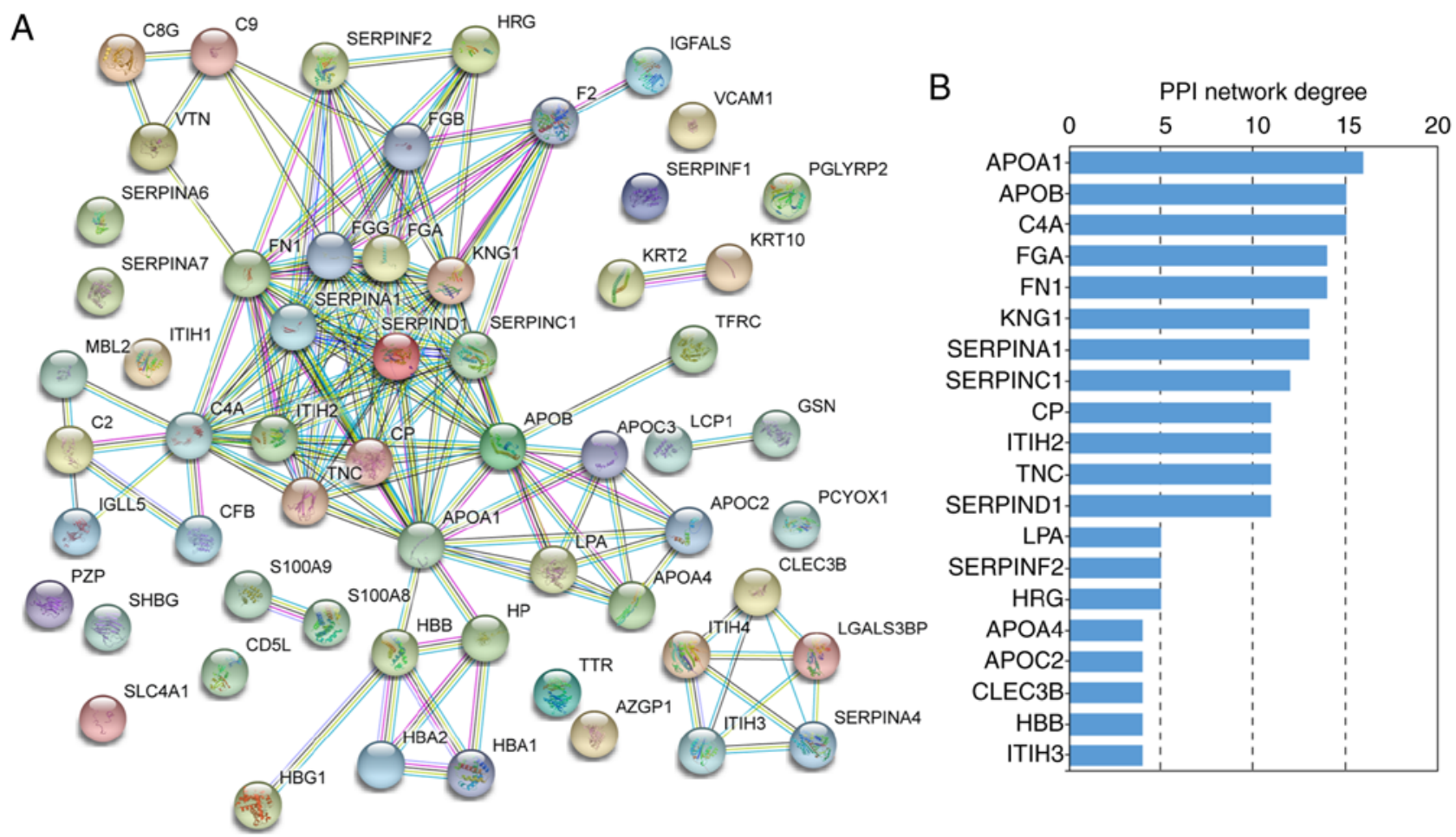

Figure 6. STRING analysis of PPI networks for the differentially expressed proteins from the +Treatment vs. control analysis. (A) PPI analysis of the 57 differentially expressed proteins from +Treatment vs. control comparison according to the STRING database using a 'highest confidence' threshold (score of 0.9). Interactions between proteins are indicated by lines. (B) PPI network degree of the top 20 differentially expressed proteins. The network degree represents the total connections of 1 protein, which can be used as a rough indication of the centrality of the protein within the PPI networks. STRING, Search Tool for the Retrieval of Interacting Genes/Proteins; PPI, Protein-protein interaction.

Validation of candidate proteins by western blot analysis and ELISA. To validate the results from the iTRAQ-based proteomic analysis, the expression levels of several candidate proteins were examined by western blot analysis and ELISA The western blot analysis results demonstrated that the expression levels of APOB and SERPINC1 were significantly downregulated in the +Treatment and -Treatment group compared with those in the control groups. Compared with the control group, SERPINA1 was significantly downregulated in the +Treatment group, but not in the -Treatment group. Compared with that in the control group, KRT1 expression was upregulated in the -Treatment group, and showed no significant difference compared with the +Treatment group. The expression of FN1 was slightly higher in the -Treatment group than in the +Treatment and control groups, but this difference was not significant (Fig. 7). The same expression changes of KRT1, APOB, FN1, SERPINC1 and SERPINA1 were further confirmed by the ELISA experiments (Fig. 8).

\section{Discussion}

In living organisms, proteins are the primary catalysts of all physiological changes (10). Protein profiling can reveal the molecular mechanisms of pathophysiological changes during disease progression and yield plausible markers that could be used for asthma diagnosis and prognosis, as well as serve as therapeutic targets. There is a vast network of complex interactions underlying disease development; no protein can account for all disease states, particularly when investigating complex diseases such as asthma. The iTRAQ technique is widely used for proteomics analysis and has the advantages of adequate sensitivity, high reproducibility and a wide linear dynamic range over traditional proteomics $(10,20)$. However, there is a lack of studies on proteomic analysis prior and subsequent to SCIT treatment in HDM-related asthma. In the present study, iTRAQ technology was used to analyze proteomic changes in HDM-related asthma. KRT1, APOB, SERPINC1 and SERPINA1 were identified as potential targets and biomarkers for the occurrence and treatment of childhood HDM-related asthma.

KRT1 is a cytoskeleton-associated type II keratin with high abundance in the epidermal cells of the spinous layer; it affects 2 important cell kinase signaling pathways associated with protein kinase $\mathrm{C}$ and SRC proto-oncogene, non-receptor tyrosine kinase (21). Studies into KRT1 have primarily focused on skin diseases such as dermatitis, ichthyosis and psoriasis (22); therefore, little is known about the association between KRT1 and HDM-related asthma. The present results indicated that KRT1 was a core protein in MCODE3 (Fig. 4B), which is mainly involved in the cellular component as a constituent of the epidermis (GO:0030280). Keratin family proteins can promote the proliferation of epithelial cells via multiple immune response pathways, and the epidermal cells are known to serve a key role in airway remodeling during asthma (23). Previous research has indicated that KRT1 was involved in the regulation of the Notch signaling pathway and affected the repair of vascular endothelial cell damage $(24,25)$. It has been identified that the specific expression of KRT1 affects complex traits (26). A previous study analyzed differential gene expression in peripheral blood mononuclear cells, 
A
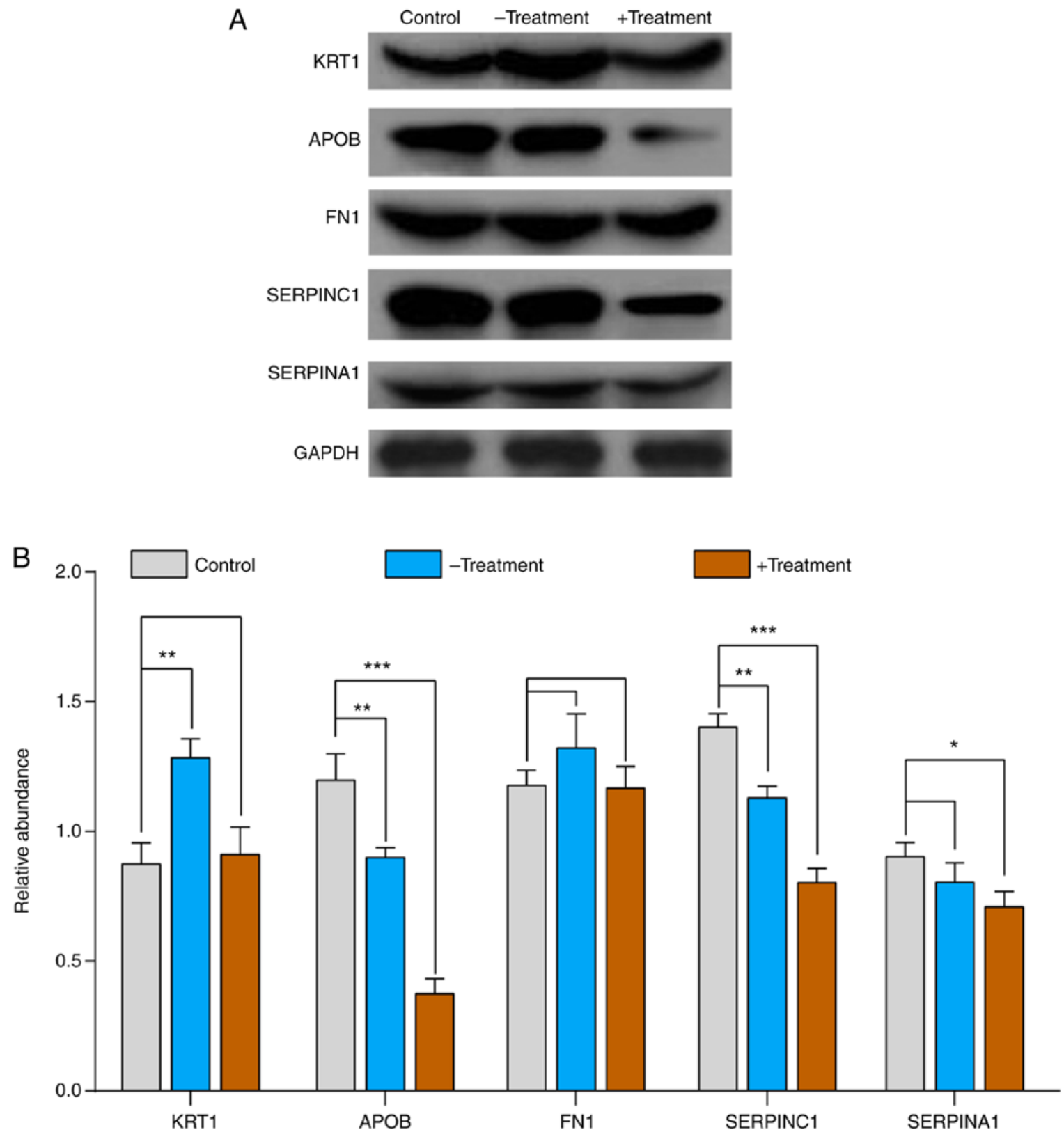

Figure 7. Validation of proteomics results by western blot analysis. (A) Western blot analysis was used for the validation of 5 proteins (KRT1, APOB, FN1, SERPINC1 and SERPINA1) from control, -Treatment and +Treatment samples; $\beta$-actin was used as an internal control. (B) Histogram of western blot analysis. The control group is shown in gray, the -Treatment group is blue and the +Treatment group is brown. Data are expressed as mean \pm standard deviation (n=25 biological replicates in each group). The multiple comparisons test of control vs. -Treatment and control vs. +Treatment are performed using analysis of variance with Dunnett's test. ${ }^{*} \mathrm{P}<0.05,{ }^{* *} \mathrm{P}<0.01$ and ${ }^{* * *} \mathrm{P}<0.001$ vs. control. KRT1, keratin 1; APOB, apolipoprotein B; FN1, fibronectin 1; SERPINC1, antithrombin III; SERPINA1, $\alpha$-1-antitrypsin.

airway brushing cells and bronchioalveolar lavage cells from asthmatic and healthy volunteers, and it was demonstrated that the major keratin family genes (KRT1, KRT4, KRT5, KRT6, KRT8 and KRT18) served important biological functions in asthma and immune responsiveness (27). In the present study, compared with the control group, the levels of KRT1 were increased three-fold in the -Treatment group, and were marginally decreased in the +Treatment group based on iTRAQ identification. This result was verified by western blot analysis and ELISA. Combined with previous studies, the present results indicated that KRT1 were strongly associated with HDM-related asthma, and may serve as a potential biomarker.
APOB is an apolipoprotein involved in the transport of lipoproteins and is associated with cholesterol metabolism (28). Previous studies investigating APOB-related genes and proteins have generally focused on diabetes, cardiovascular disease and rheumatoid diseases (28-30). It has been reported that APOB has a role in the regulation of cellular inflammation and cardiovascular inflammation after viral infections, as well as having a role in the inflammatory response during the progression of rheumatoid arthritis $(31,32)$. In the current study, APOB was identified to be associated with the most significantly enriched biological process term regarding acute inflammatory responses, and was predicted be a major hub protein in the PPI network. However, the role of APOB in the 


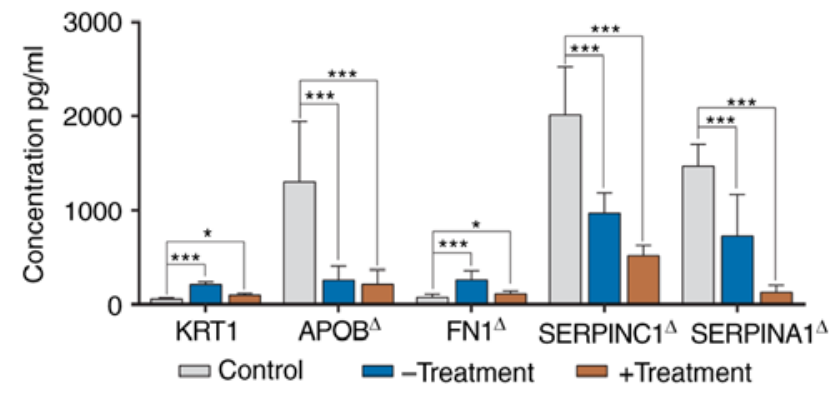

Figure 8. Validation of the 5 candidate proteins (KRT1, APOB, FN1, SERPINC1 and SERPINA1) by ELISA in the control children, and -Treatment and +Treatment patients. The control group is shown in gray, the -Treatment in blue and the + Treatment in brown. Data are expressed as mean \pm standard deviation ( $\mathrm{n}=25$ biological replicates in each group). The multiple comparisons test of control vs. -Treatment and control vs. +Treatment are performed using ANOVA with Dunnett or Welch with Dunnett's T3 test based on the result of variance homogeneity. Except for KRT1 (ANOVA statistics), the variances of APOB, FN1, SERPINC1 and SERPINA1 were not homogenous. ${ }^{\Delta}$ Data were analyzed using a Welch test. ${ }^{*} \mathrm{P}<0.05$ and ${ }^{* * * *} \mathrm{P}<0.001$ vs. control. KRT1, keratin 1; APOB, apolipoprotein B; FN1, fibronectin 1; SERPINC1, antithrombin III; SERPINA1, $\alpha$-1-antitrypsin; ANOVA, analysis of variance.

molecular pathogenesis of asthma has been disputed in several studies (33-37). A previous study found no association between the levels of APOB in plasma and asthma symptoms (33). By contrast, subsequent studies have revealed a significant positive correlation between APOB and platelet activating factor (PAF) in allergic reactions, and concluded that PAF is a key pathogenic regulator of asthma, whose stability is affected by APOB $(34,35)$. Furthermore, it has been suggested that the expression of APOB in the asthma and control groups may be affected by polymorphisms in the pro-inflammatory gene ORMDL sphingolipid biosynthesis regulator 3 (36). In addition, some researchers identified that the expression level of APOB was negatively correlated with the severity index of asthma $\left(\mathrm{FEV}_{1 \%}\right)(37)$. Therefore, APOB may serve as a candidate asthma-associated target or biomarker and may be involved in the pathological progression of asthma. However, little attention has been given to determining how APOB expression may change in asthma following SCIT treatment. In the present study, it was identified that the expression of APOB was downregulated in the -Treatment and +Treatment groups compared with that in the control group. Combined with the results of previous studies, it was proposed that APOB could be a potential target for HDM-related asthma.

FN1 is a fibroadenoma protein involved in cell adhesion and migration; it can affect wound healing and host immune processes. Studies have shown that the FN1 gene is differentially expressed in asthma caused by viral infection (38). Yang et al (39) identified that WISP1 initiates and propagates the pathological airway remodeling process of asthma by inducing fibroblast proliferation and ECM deposition in mouse and HFL-1 cell models, and that FN1 may be involved in this regulatory mechanism. A previous study has also identified that interleukin 33 promotes the pathological process of asthmatic airway remodeling by inducing the over-expression of FN1 in HLF-1 cells (40). In the present study it was identified that FN1 was upregulated in HDM-related asthma groups (-Treatment and +Treatment) compared with the control group by iTRAQ analysis and ELISA; however, there was no significant difference in FN1 expression based on the data from the western blot analysis. This suggested that FN1 may exhibit high heterogeneity in HDM-associated asthma subjects, but the role of FN1 in asthma remains to be clarified.

SERPINC1 belongs to the serine protease family encoding a plasma protease inhibitor, which serves a key role in coagulation regulation. In the past decade, considerable research has identified that genetic variants of SERPINC1 were associated with a risk of thrombosis (41-44). Furthermore, an epidemiological study suggested that patients with asthma have a significantly increased risk of venous thromboembolism and pulmonary embolism (45). A further study has shown that the coagulation and fibrinolysis parameters of endogenous thrombin potential plasminogen activator inhibitor type 1 and D-dimer, and von Willebrand factor levels increased with increasing asthma severity (46). The previous study also indicated a significant positive correlation between the prothrombotic state and disease severity in patients with asthma (46). These results explain the increased risk of venous thromboembolism and pulmonary embolism in patients with asthma. Few studies have focused on the role of SERPINC1 in asthma; therefore, it remains unknown whether the increased risk of venous thrombosis in patients with asthma is associated with SERPINC1. Recently, a large-scale epigenome-wide association study found that SERPINC1 methylation was strongly associated with asthma in children (47). These results suggested that SERPINC1 may serve as a sympathetic molecule for the development of asthma, vascular thrombosis and pulmonary embolism; thus, it is an important finding with academic value. In the current study, compared with the control group, the levels of SERPINC1 were significantly decreased in the +Treatment asthma group based on iTRAQ analysis, western blot analysis and ELISA verification. To the best of our knowledge, the present study provided the first demonstration that the protein expression of SERPINC1 was associated with HDM-related asthma, which may provide novel insights into its pathogenesis.

SERPINA1 encodes a serine proteinase inhibitor $\alpha-1$ antitrypsin (ATT) that inactivates elastase, proteinase 3 and cathepsin $\mathrm{G}$ in neutrophils, protecting the lower respiratory tract and lungs against attacks by these proteases $(48,49)$. ATT deficiencies have been associated with a number of diseases, such as pulmonary emphysema, chronic obstructive pulmonary disease and cystic fibrosis liver disease, but its role in the development of asthma remains controversial (48-51). Several studies have suggested that SERPINA1 mutations resulted in a deficiency in ATT, leading to allergic asthma (52-54). In the present study, it was identified that SERPINA1 was marginally downregulated in the +Treatment group compared with the control group, confirming that SERPINA1 was associated with the development of HDM-related asthma.

There were certain limitations to the present study. Serum samples were used instead of blood cells to perform proteome analysis, as the protein composition of blood cells is more complex; furthermore, there are several different types of blood cells, each of which may be associated with the pathophysiology of asthma. Therefore, this limits the comparisons of the present study with previous studies that investigated blood cells samples. In addition, 5 samples from each group were mixed into a pooled group sample in iTRAQ, which can lead to 
more significant systematic errors. To address this problem, an independent cohort was used for validation; however, the role of KRT1, APOB, SERPINC1 and SERPINA1 in HDM-related asthma requires further validation in a broader cohort.

In conclusion, 72 differentially expressed proteins that serve a role in the progression of HDM-related asthma were identified. A panel of 4 proteins (KRT1, APOB, SERPINC1 and SERPINA1), which were validated as differentially expressed using western blot analysis, show potential as biomarkers for HDM-related asthma. Functional classification and PPI network analysis of the identified proteins provided insights into the complex pathogenesis of asthma. Moreover, the role of SERPINC1 in HDM-related asthma will improve the understanding of the progression of asthma. In future studies, further validation of the roles of KRT1, APOB, SERPINC1 and SERPINA1 in a larger HDM-related asthma cohort is required to confirm these data.

\section{Acknowledgements}

The authors would like to thank Professor Yin Guan (Southern Medical University, Guangzhou, China) for her help with the statistical analysis.

\section{Funding}

The present study was supported by the Foundation of Hunan Double First-rate Discipline Construction Projects of Bioengineering, and the Major Project for Guangzhou Collaborative Innovation of Industry-University-Research (grant no. 201704020196 to RH).

\section{Availability of data and materials}

The datasets used and/or analyzed during the current study are available from the corresponding author on reasonable request.

\section{Authors' contributions}

JB and SPL conceived and designed the study. WL and LC prepared the samples and completed the molecular biology experiments. XJW, JYZ and RH analyzed the data. SPL and XJW drafted the manuscript. All authors read and approved the final manuscript.

\section{Ethics approval and consent to participate}

This study was approved by the Ethics Committee of Foshan Maternal and Children's Hospital Affiliated to Southern Medical University (policy no. FSFY-MEC-2019-013). Written informed consent was obtained from the guardians of each participant.

\section{Patient consent for publication}

The written informed consent for the publication was obtained from the guardians of each participant.

\section{Competing interests}

The authors declare that they have no competing interests.

\section{References}

1. Global Initiative for Asthma: Global Strategy for Asthma Management and Prevention, 2018. Available from: http://www. ginasthma.org.

2. Asthma Group, Society of Respiratory Diseases, Chinese Medical Association: Guidelines for the prevention and treatment of bronchial asthma. Chin J Tuberculosis Respir 39: 675-697, 2016.

3. Bateman ED, Hurd SS, Barnes PJ, Bousquet J, Drazen JM, FitzGerald JM, Gibson P, Ohta K, O'Byrne P, Pedersen SE, et al: Global strategy for asthma management and prevention: GINA executive summary. Eur Respir J 31: 143-178, 2008.

4. Huang FL, Liao EC and Yu SJ: House dust mite allergy: Its innate immune response and immunotherapy. Immunobiology 223: 300-302, 2018

5. Eyerich S, Metz M, Bossios A and Eyerich K: New biological treatments for asthma and skin allergies. Allergy 75: 546-560, 2020.

6. Pfaar O, Lou H, Zhang Y, Klimek L and Zhang L: Recent developments and highlights in allergen immunotherapy. Allergy 73: 2274-2289, 2018.

7. Shamji M, Kappen J, Akdis M, Jensen-Jarolim E, Knol EF, Kleine-Tebbe J, Bohle B, Chaker AM, Till SJ, Valenta R, et al: Biomarkers for monitoring clinical efficacy of allergen immunotherapy for allergic rhinoconjunctivitis and allergic asthma: An EAACI position paper. Allergy 72: 1156-1173, 2017.

8. Keles S, Karakoc-Aydiner E, Ozen A, Izgi AG, Tevetoglu A, Akkoc T, Bahceciler NN and Barlan I: A novel approach in allergen-specific immunotherapy: Combination of sublingual and subcutaneous routes. J Allergy Clin Immunol 128: 808-815 e7, 2011.

9. Celis JE, Gromov P, Cabezon T, Moreira JMA, Ambartsumian N, Sandelin K, Rank F and Gromova I: Proteomic characterization of the interstitial fluid perfusing the breast tumor microenvironment: A novel resource for biomarker and therapeutic target discovery. Mol Cell Proteomics 3: 327-344, 2004.

10. Ray S, Reddy PJ, Jain R, Gollapalli K, Moiyadi A and Srivastava S: Proteomic technologies for the identification of disease biomarkers in serum: Advances and challenges ahead. Proteomics 11: 2139-2161, 2011.

11. Thomas PD, Campbell MJ, Kejariwal A, Mi H, Karlak B, Daverman R, Diemer K, Muruganujan A and Narechania A: PANTHER: A library of protein families and subfamilies indexed by function. Genome Res 13: 2129-2141, 2003.

12. Mi H, Muruganujan A and Thomas PD: PANTHER in 2013: Modeling the evolution of gene function, and other gene attributes, in the context of phylogenetic trees. Nucleic Acids Res 41: D377-D386, 2013.

13. Mi H, Muruganujan A, Ebert D, Huang $X$ and Thomas PD: PANTHER version 14: More genomes, a new PANTHER GO-slim and improvements in enrichment analysis tools. Nucleic Acids Res 47: D419-D426, 2019.

14. Tripathi S, Pohl M, Zhou Y, Rodriguez-Frandsen A, Wang G, Stein DA, Moulton HM, DeJesus P, Che J, Mulder LCF, et al: Meta- and orthogonal integration of influenza 'OMICs' data defines a role for UBR4 in virus budding. Cell Host Microbe 18: 723-735, 2015.

15. Kanehisa M and Goto S: KEGG: Kyoto encyclopedia of genes and genomes. Nucleic Acids Res 28: 27-30, 2000.

16. Kanehisa M, Sato Y, Furumichi M, Morishima K and Tanabe M: New approach for understanding genome variations in KEGG. Nucleic Acids Res 47: D590-D595, 2019.

17. Kanehisa M: Toward understanding the origin and evolution of cellular organisms. Protein Sci 28: 1947-1951, 2019.

18. Shannon P, Markiel A, Ozier O, Baliga NS, Wang JT, Ramage D, Amin N, Schwikowski B and Ideker T: Cytoscape: A software environment for integrated models of biomolecular interaction networks. Genome Res 13: 2498-2504, 2003.

19. Szklarczyk D, Gable AL, Lyon D, Junge A, Wyder S, Huerta-Cepas J, Simonovic M, Doncheva NT, Morris JH, Bork P, et al: STRING v11: Protein-protein association networks with increased coverage, supporting functional discovery in genome-wide experimental datasets. Nucleic Acids Res 47: D607-D613, 2019.

20. Aggarwal K, Choe LH and Lee KH: Shotgun proteomics using the iTRAQ isobaric tags. Brief Funct Genomics Proteomic 5: 112-120, 2006.

21. Roth W, Kumar V, Beer HD, Richter M, Wohlenberg C, Reuter U, Thiering S, Staratschek-Jox A, Hofmann A, Kreusch F, et al: Keratin 1 maintains skin integrity and participates in an inflammatory network in skin through interleukin-18. J Cell Sci 125: 5269-5279, 2012. 
22. Choate KA, Lu Y, Zhou J, Elias PM, Zaidi S, Paller AS, Farhi A, Nelson-Williams C, Crumrine D, Milstone LM and Lifton RP: Frequent somatic reversion of KRT1 mutations in ichthyosis with confetti. J Clin Invest 125: 1703-1707, 2015.

23. Depianto D, Kerns M, Dlugosz A and Coulombe PA: Keratin 17 promotes epithelial proliferation and tumor growth by polarizing the immune response in skin. Nat Genet 42: 910-914, 2010.

24. Gao Z, Ji X, Gu J, Wang XY, Ding L and Zhang H: microRNA-107 protects against inflammation and endoplasmic reticulum stress of vascular endothelial cells via KRT1-dependent Notch signaling pathway in a mouse model of coronary atherosclerosis. J Cell Physiol 234: 12029-12041, 2019.

25. Fang HC, Wu BQ, Hao YL, Luo Y, Zhao HL, Zhang WY, Zhang ZL, Liang JJ, Liu W and Chen XH: KRT1 gene silencing ameliorates myocardial ischemia-reperfusion injury via the activation of the Notch signaling pathway in mouse models. J Cell Physiol 234: 3634-3646, 2019.

26. Tao H, Cox D and Frazer K: Allele-specific KRT1 expression is a complex trait. PLoS Genet 2: e93, 2006.

27. Gao P, Grigoryev D, Breslin L, Cheadle C, Mathias RA, Beaty TH, Togias A and Barnes K: Keratins: Important candidate genes for asthma and immune responsiveness to cockroach. J Allergy Clin Immunol 119 (Suppl): S176, 2007.

28. Whitfield AJ, Barrett PH, Van Bockxmeer FM and Burnett JR: Lipid disorders and mutations in the APOB gene. Clin Chem 50: 1725-1732, 2004.

29. Duvillard L, Pont F, Florentin E, Galland-Jos C, Gambert P and Vergès B: Metabolic abnormalities of apolipoprotein B-containing lipoproteins in non-insulin-dependent diabetes: A stable isotope kinetic study. Eur J Clin Invest 30: 685-694, 2000

30. Walldius $\mathrm{G}$ and Jungner I: The apoB/apoA-I ratio: A strong, new risk factor for cardiovascular disease and a target for lipid-lowering therapy-a review of the evidence. J Intern Med 259: 493-519, 2006.

31. Tort O, Escribà T, Egaña-Gorroño L, de Lazzari E, Cofan M, Fernandez E, Gatell JM, Martinez E, Garcia F and Arnedo M: Cholesterol efflux responds to viral load and CD4 counts in $\mathrm{HIV+}$ patients and is dampened in HIV exposed. J Lipid Res 59: 2108-2115, 2018.

32. Lee J, Kang M, Choi J, Park JS, Park JK, Lee EY, Lee EB, Pap T, Yi EC and Song YW: Apolipoprotein B binds to enolase-1 and aggravates inflammation in rheumatoid arthritis. Ann Rheum Dis 77: 1480-1489, 2018.

33. Nagel G, Weiland S, Rapp K, Link B, Zoellner I and Koenig W: Association of apolipoproteins with symptoms of asthma and atopy among schoolchildren. Int Arch Allergy Immunol 149: 259-266, 2009

34. Pettersson ME, Koppelman GH, Flokstra-de Blok BM, van Ginkel CD, Roozendaal C, Muller-Kobold AC, Kollen BJ and Dubois AEJ: Apolipoprotein B: A possible new biomarker for anaphylaxis. Ann Allergy Asthma Immunol 118: 515-516, 2017.

35. Perelman B, Adil A and Vadas P: Relationship between platelet activating factor acetylhydrolase activity and apolipoprotein $\mathrm{B}$ levels in patients with peanut allergy. Allergy Asthma Clin Immunol 10: 20, 2014.

36. Wang BJ, Wang GL, Chen DH, Wang WX, Huang J, Rong JY, Liu XT and Yang S: Association of ORMDL3 single nucleotide polymorphisms with lysophosphatidylcholine and apolipoprotein $\mathrm{B}$ levels in children with asthma. Zhongguo Dang Dai Er Ke Za Zhi 17: 241-244, 2015 (In Chinese).

37. Barochia AV, Kaler M, Cuento RA, Gordon EM, Weir NA, Sampson M,Fontana JR, MacDonald S, Moss J,Manganiello V, et al: Serum apolipoprotein A-I and large high-density lipoprotein particles are positively correlated with FEV1 in atopic asthma. Am J Respir Crit Care Med 191: 990-1000, 2015.

38. Bochkov Y, Hanson K, Keles S, Brockman-Schneider RA, Jarjour NN and Gern JE: Rhinovirus-induced modulation of gene expression in bronchial epithelial cells from subjects with asthma. Mucosal Immunol 3: 69-80, 2010.
39. Yang M, Zhao X, Liu Y, Tian Y, Ran X and Jiang Y: A role for WNT1-inducible signaling protein-1 in airway remodeling in a rat asthma model. Int Immunopharmacol 17: 350-357, 2013.

40. Guo Z, Wu J, Zhao J, Liu F, Chen Y, Bi L and Dong L: IL-33/ST2 promotes airway remodeling in asthma by activating the expression of fibronectin 1 and type 1 collagen in human lung fibroblasts. Xi Bao Yu Fen Zi Mian Yi Xue Za Zhi 30: 975-979, 2014 (In Chinese).

41. Bezemer ID, Bare LA, Doggen CJ, Arellano AR, Tong C, Rowland CM, Catanese J, Young BA, Reitsma PH, Devlin JJ and Rosendaal FR: Gene variants associated with deep vein thrombosis. JAMA 299: 1306-1314, 2008.

42. de Haan H, Bezemer I, Doggen C, Cessie SL, Reitsma PH, Arellano AR, Tong CH, Devlin JJ, Bare LA, Rosendaal FR and Vossen CY: Multiple SNP testing improves risk prediction of first venous thrombosis. Blood 120: 656-663, 2012.

43. Luxembourg B, Pavlova A, Geisen C, Spannagl M, Bergmann F, Krause M, Alesci S, Seifried E and Lindhoff-Last E: Impact of the type of SERPINC1 mutation and subtype of antithrombin deficiency on the thrombotic phenotype in hereditary antithrombin deficiency. Thromb Haemost 111: 249-257, 2014.

44. Rosendaal F: Causes of venous thrombosis. Thromb J 14 (Suppl 1): S24, 2016.

45. Majoor CJ, Kamphuisen PW, Zwinderman AH, Ten Brinke A, Amelink M, Rijssenbeek-Nouwens L, Sterk PJ, Büller HR and Bel EH: Risk of deep vein thrombosis and pulmonary embolism in asthma. Eur Respir J 42: 655-661, 2013.

46. Sneeboer MMS, Majoor CJ, de Kievit A, Meijers JCM, van der Poll T, Kamphuisen PW and Bel EH: Prothrombotic state in patients with severe and prednisolone-dependent asthma. J Allergy Clin Immunol 137: 1727-1732, 2016.

47. Xu CJ, Söderhäll C, Bustamante M, Baïz N, Gruzieva O, Gehring U, Mason D, Chatzi L, Basterrechea M, Llop S, et al: DNA methylation in childhood asthma: An epigenome-wide meta-analysis. Lancet Respir Med 6: 379-388, 2018.

48. Cosio MG, Bazzan E, Rigobello $\mathrm{C}$, Tinè $\mathrm{M}$, Turato $\mathrm{G}$, Baraldo $\mathrm{S}$ and Saetta M: Alpha-1 antitrypsin deficiency: Beyond the protease/antiprotease paradigm. Ann Am Thorac Soc 4 (Suppl 13): S305-S310, 2016.

49. McCarthy C, Reeves EP and McElvaney NG: The role of neutrophils in alpha-1 antitrypsin deficiency. Ann Am Thorac Soc 4 (Suppl 13): S297-S304, 2016.

50. De Serres F and Blanco I: Role of alpha-1 antitrypsin in human health and disease. J Intern Med 276: 311-335, 2014.

51. Janciauskiene S and Welte T: Well-known and less well-known functions of alpha-1 antitrypsin. Its role in chronic obstructive pulmonary disease and other disease developments. Ann Am Thorac Soc 4 (Suppl 13): S280-S288, 2016.

52. Bragina EY, Goncharova IA, Garaeva AF, Nemerov EV, Babovskaya AA, Karpov AB, Semenova YV, Zhalsanova IZ, Gomboeva DE, Saik OV, et al: Molecular relationships between bronchial asthma and hypertension as comorbid diseases. J Integr Bioinform 15: 20180052, 2018.

53. Suárez-Lorenzo I, de Castro FR, Cruz-Niesvaara D, Herrera-Ramos E, Rodríguez-Gallego $\mathrm{C}$ and Carrillo-Diaz T: Alpha 1 antitrypsin distribution in an allergic asthmatic population sensitized to house dust mites. Clin Transl Allergy 8: 44, 2018.

54. Hernández-Pérez JM, Ramos-Díaz R and Pérez JA: Identification of a new defective SERPINA1 allele ( PI*Z la palma) encoding an alpha-1-antitrypsin with altered glycosylation pattern. Respir Med 131: 114-117, 2017.

This work is licensed under a Creative Commons Attribution-NonCommercial-NoDerivatives 4.0 International (CC BY-NC-ND 4.0) License. 